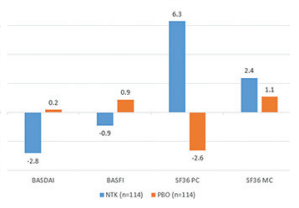

Figure 2. Change in BASDAI, BASFI and QoL at Wk 16 (means)

Disclosure of Interests: Inna Gaydukova Grant/research support from: JSC BIOCAD, Speakers bureau: paiment from Pfizer, Novartis, Abbvie, Biocad, Selgene, MSD, Sanofy does not exceed 10000 euros, V Mazurov Grant/research support from: JSC BIOCAD, Shandor Erdes Grant/research support from: JSC BIOCAD, Speakers bureau: JSC BIOCAD, Tatiana Dubinina: None declared, Olga Nesmeyanova Grant/ research support from: JSC BIOCAD, Elena llivanova Grant/research support from: JSC BIOCAD, Alena Kundzer: None declared, Nikolaj Soroka: None declared, Ekaterina Dokukina Employee of: JSC BIOCAD, Anna Eremeeva Grant/research support from: JSC BIOCAD, Ekaterina Chernyaeva Employee of: JSC BIOCAD, Roman Ivanov Employee of: JSC BIOCAD

DOI: 10.1136/annrheumdis-2019-eular.6791

\section{FRI0392 EVIDENCE BASED RECOMMENDATIONS FOR THE MANAGEMENT OF ENTEROPATHIC ARTHRITIS: A RHEUMATOLOGY, GASTROENTEROLOGY COLLABORATIVE INITIATIVE}

Gulen Hatemi ${ }^{1}$, Servet Akar $^{2}$, Hale Akpinar ${ }^{3}$, Pamir Atagündüz $z^{4}$, Goksel Bengi ${ }^{3}$, Gerçek Can ${ }^{5}$, Aykut Ferhat Celik ${ }^{6}$, Sinem Nihal Esatoglu', Önay Gerçik ${ }^{2}$, Hulya Hamzaoglư ${ }^{7}$, Murat Inanc ${ }^{8}$, Gokhan Kabacam ${ }^{9}$, Ismail Hakkı Kalkan ${ }^{10}$, Levent Kılıç ${ }^{11}$, Fatos Onen ${ }^{5}$, Ahmet Tezel ${ }^{12}$, Murat Toruner ${ }^{13}$, Sedat Kiraz ${ }^{11}$. ${ }^{1}$ Istanbul University-Cerrahpasa, Cerrahpasa Medical Faculty, Department of Internal Medicine, Division of Rheumatology, Istanbul, Turkey; ${ }^{2}$ Izmir Katip Celebi University, Faculty of Medicine, Department of Internal Medicine, Division of Rheumatology, Izmir, Turkey; ${ }^{3}$ Dokuz Eylul University, Faculty of Medicine, Department of Internal Medicine, Division of Gastroenterology, Izmir, Turkey; ${ }^{4}$ Marmara University Pendik Research and Educational Hospital, Department of Internal Medicine, Division of Gastroenterology, Istanbul, Turkey, ${ }^{5}$ Dokuz Eylul University, Faculty of Medicine, Department of Internal Medicine, Division of Rheumatology, Izmir, Turkey; ${ }^{6}$ Istanbul University-Cerrahpasa, Cerrahpasa Medical Faculty, Department of Internal Medicine, Division of Gastroenterology, Istanbul, Turkey, ${ }^{7}$ Acibadem University, Acibadem Fulya Hospital, Department of Gastroenterology, Istanbul, Turkey, ${ }^{8}$ Istanbul University, Istanbul Faculty of Medicine, Department of Internal Medicine, Division of Rheumatology, Istanbul, Turkey, ${ }^{9}$ Ankara Guven Hospital, Department of Gastroenterology, Ankara, Turkey; ${ }^{10}$ Türkiye Yüksek htisas Training and Research Hospital, Department of Gastroenterology, Ankara, Turkey, ${ }^{11}$ Hacettepe University Faculty of Medicine, Department of Internal Medicine, Division of Rheumatology, Ankara, Turkey, ${ }^{12}$ Abant izzet Baysal University School of Medicine, Department of Gastroenterology, Bolu, Turkey, ${ }^{13}$ Ankara University School of Medicin, Department of Gastroenterology, Ankara, Turkey

Background: Management of enteropathic arthritis may be challenging due to differences in treatment response of inflammatory bowel diseases and arthritis to different therapeutic modalities, which may even cause worsening of some manifestations while improving others. Enteropathic arthritis was not addressed in the management recommendations for spondyloarthritis.

Objectives: The aim of this project was to develop a set of evidence based recommendations for the management of patients with enteropathic arthritis.

Methods: A task force was formed that included ten rheumatologists and 8 gastroenterologists. Research questions were determined using a Delphi approach. A systematic literature search, data extraction, and statistical analyses were performed according to a pre-specified protocol. Studies that assessed the efficacy of an intervention on inflammatory bowel disease related outcomes and/or spondyloarthritis relates outcomes in patients with enteropathic arthritis were included. Risk ratios were calculated for binary outcomes and mean difference for continuous outcomes, whenever possible. Results of the systematic literature review were presented to the experts and recommendations were formulated after thorough discussions and voting.

Results: A total of 4 overarching principles and 10 recommendations were formulated. The recommendations addressed the use of NSAIDs, corticosteroids, sulfasalazine and 5-ASA derivatives, TNF inhibitors, tofacitinib, secukinumab, ustekinumab and vedolizumab among patients with active inflammatory bowel disease, active arthritis, active disease regarding both inflammatory bowel disease and arthritis, and among patients in remission. Final voting showed good agreement among the group on all recommendations.

Conclusion: These recommendations are intended to help rheumatologists, gastroenterologists and other clinicians dealing with enteropathic arthritis and to point out to the shortcomings of the available data on the management of this challenging condition.

Disclosure of Interests: Gulen Hatemi Consultant for: Abbvie, Amgen, BMS, Janssen, MSD, Pfizer, UCB, Speakers bureau: Abbvie, Amgen, BMS, Jansen, MSD, Pfizer, UCB, Servet Akar Grant/research support from: MSD, Abbvie, Roche, UCB, Novartis, Pfizer, Amgen, Consultant for: MSD, Abbvie, Roche, UCB, Novartis, Pfizer, Amgen, Speakers bureau: Pfizer, Hale Akpinar: None declared, Pamir Atagündüz: None declared, Goksel Bengi: None declared, Gerçek Can: None declared, Aykut Ferhat Celik: None declared, Sinem Nihal Esatoglu: None declared, Önay Gerçik: None declared, Hulya Hamzaoglu: None declared, Murat Inanc: None declared, Gokhan Kabacam: None declared, Ismail Hakkı Kalkan: None declared, Levent Kilıç: None declared, Fatos Onen: None declared, Ahmet Tezel: None declared, Murat Toruner: None declared, Sedat Kiraz: None declared

DOI: 10.1136/annrheumdis-2019-eular.7930

\section{FRI0393 PATIENTS' SATISFACTION AND PREFERENCES TOWARDS SUPERVISED GROUP EXERCISE FOR PEOPLE WITH AXIAL SPONDYLOARTHRITIS}

Bas Hilberdink ${ }^{1}$, Florus van der Giesen ${ }^{2}$, Thea Vliet Vlieland ${ }^{1}$, Floris A. van Gaalen², Salima van Weely ${ }^{1}$. $^{1}$ Leiden University Medical Center, Orthopaedics, Rehabilitation and Physical Therapy, Leiden, Netherlands; ${ }^{2}$ Leiden University Medical Center, Rheumatology, Leiden, Netherlands

Background: Supervised group exercise (SGE) is proven effective in patients with axial spondyloarthritis (axSpA), but its contents and dosage do not always comply with current scientific insights. ${ }^{1}$

In particular vigorous intensity cardiorespiratory exercise is considered to be an important element of exercise programs in axSpA. ${ }^{2}$ For successful implementation of any adjustments, axSpA patients' satisfaction and preferences towards SGE needs to be determined.

Objectives: This study aimed to describe axSpA patients' satisfaction with current SGE and perspective on potential, evidence-based SGE enhancements.

Methods: AxSpA patients participating in SGE in four regions in the Netherlands $(n=118)$ completed a survey on their satisfaction with features of current SGE (eight questions, 3-point Likert scale, and one overall grade, 11-point scale) and their perspective on introducing appropriately dosed cardiorespiratory and strengthening exercise, monitoring exercise intensity, periodic (re)assessments, patient education and supervision by physical therapists with specific expertise (four dichotomous questions and one 5-point Likert scale). In addition, sociodemographic and disease characteristics were recorded.

Results: The patients' mean age was 60 years (SD \pm 12 ), 64\% was male and they participated in SGE for 25 years $(S D \pm 14)$ on average. The SGE programs in the four regions all took place once weekly between 90 to 135 minutes and all consisted of mobility exercises, sports activities and hydrotherapy. Two regions also focused on strengthening and only one specifically addressed cardiorespiratory exercise.

Most patients were satisfied with the current total intensity $(n=84 / 112$, $75 \%)$, duration $(n=93 / 111,84 \%)$ and load $(n=89 / 117,76 \%)$ of the program and the proportion of mobility $(n=102 / 114,90 \%)$, strengthening $(n=90 / 115,78 \%)$ and cardiorespiratory exercise $(n=82 / 114,72 \%)$. The median overall grade of the program was a 7 (IQR=7-8). Most patients agreed with the implementation of more frequent (home) exercise $(n=73$ / $117,62 \%)$, heartrate monitoring $(n=97 / 117,83 \%)$ and periodic (re)assessments $(n=97 / 118,82 \%)$, whereas $50 \%$ agreed with the addition of structured patient education $(n=37 / 74)$. The majority found supervision by therapists specialized in axSpA highly important $(n=105 / 118,89 \%)$.

Conclusion: The majority of axSpA-specific SGE participants was satisfied with current SGE, but also agreed with enhancements in line with scientific evidence. The high satisfaction levels with the amount of cardiorespiratory exercise, despite only being targeted in one SGE region, suggests a knowledge gap regarding its (health) benefits. Current satisfaction levels indicate that a planned implementation strategy, including education and addressing potential barriers and facilitators for the uptake of enhancements, is warranted. 